\title{
Arbaclofen Placarbil Decreases Reflux With Good Tolerability in Patients With Gastroesophageal Reflux Disease
}

(Am J Gastroenterol 2010;105:1266-1275)

Gwang Ha Kim, MD, PhD

Department of Internal Medicine, Pusan National University School of Medicine, Busan, Korea

\section{Summary}

Arbaclofen placarbil (AP), previously designated as XP19986, is a novel transported prodrug of the active R-isomer of baclofen, which overcomes the pharmacokinetic limitations of racemic baclofen. ${ }^{1} \mathrm{AP}$ is efficiently absorbed by high-capacity active transport pathways expressed throughout the gastrointestinal (GI) tract, and rapidly metabolized to release R-baclofen after the absorption.

This multicenter, randomized, double-blind, crossover study comparing single doses of AP with placebo was conducted to assess the efficacy and safety of AP for decreasing meal-induced reflux episodes in patients with gastroesophageal reflux disease (GERD). ${ }^{2}$

Different patients were enrolled at each of 4 escalating AP doses: 10, 20, 40 and $60 \mathrm{mg}$. A total of 50 patients were treated and the efficacy analysis included 44 patients who completed the protocol and had technically satisfactory impedance $/ \mathrm{pH}$ data. There was a statistically significant decrease in reflux episodes over 12 hours after treatment with AP compared with placebo ( $\mathrm{p}$ $=0.01)$. The mean $\pm \mathrm{SD}$ number of reflux episodes over 12 hours after AP treatment was $50.5 \pm 27.2$, with a mean reduction of $10.4 \pm 23.9$ episodes (17\%) compared with placebo (60.9 \pm 35.3). Only acid reflux episodes were statistically decreased after treatment with AP. Heartburn events associated with reflux were reduced during treatment with AP compared with placebo $(12.9 \pm 11.9$ vs $16.7 \pm 18.2, \mathrm{p}=0.03)$. AP seemed to be the most efficacious in the $60 \mathrm{mg}$ dose group, and was well tolerated at all dose levels.

\section{Comment}

Up to $40 \%$ of GERD patients fail to completely respond symptomatically to standard proton pump inhibitor doses. ${ }^{3}$ GERD is commonly associated with transient lower esophageal sphincter relaxations (TLESRs), which are relaxations not related to swallowing. ${ }^{4}$ TLESRs are triggered by gastric distension, mediated by mechanosensitive vagal afferent neurons terminating in the brainstem, resulting in a vago-vagal reflex motor pattern that also involves inhibition of the crural diaphragm and longitudinal muscle contractions in the distal esophagus. ${ }^{5}$

Racemic baclofen, an agonist of $\gamma$-aminobutyric acid receptor, has been shown to decrease reflux through the inhibition of

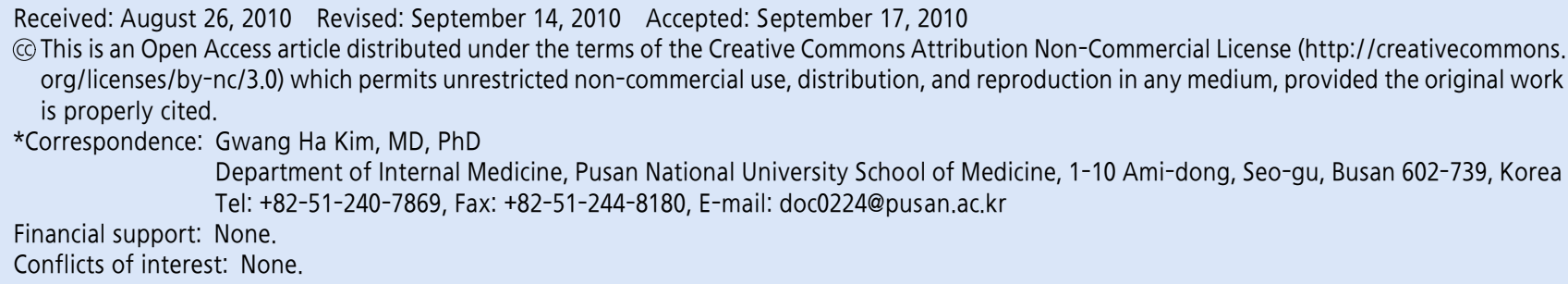


TLESRs and the augmentation of lower esophageal sphincter pressure. A study using impedance/pH found that $40 \mathrm{mg}$ of baclofen significantly reduced total reflux episodes and associated GERD symptoms over a period of 2 hours following a refluxogenic meal given 1 hour after the treatment. ${ }^{6}$ Another study showed that administration of baclofen, $10 \mathrm{mg}, 4$ times a day (q.i.d.), decreased reflux episodes by $51 \%$ over 24 hours. ${ }^{7}$ In the same study, the reduction in reflux episodes and reflux symptoms was maintained after 4 weeks of treatment with baclofen, $10 \mathrm{mg}$ q.i.d., compared with placebo. This observation suggests that the reduction in reflux episodes and associated symptoms after a single dose of AP may also result in clinically relevant benefits through 4 weeks of treatment in subsequent studies.

However, the limitations of baclofen include a short half-life requiring frequent dosing, and adverse effects including sedation, dizziness, nausea and fatigue. ${ }^{8}$ Orally administered baclofen is absorbed from the lumen of the GI tract through an active transport system found predominantly in the proximal small bowel. However, the lack of significant absorption in the lower intestine has precluded the development of a sustained-release formulation.

$\mathrm{AP}$ is developed in a sustained-release formulation, allowing for decreased dosing frequency with reduced fluctuation in R-baclofen exposure due to its absorption in the lower GI tract. ${ }^{9}$ This may lead to improved efficacy and a decreased incidence of adverse effects compared with racemic baclofen.

Therefore, this present study assessed the efficacy of AP for decreasing the total number of reflux episodes in patients with GERD, in whom substantial baseline incidence of meal-induced reflux was confirmed by impedance/pH measurements. The authors found that AP significantly reduced the total number of reflux episodes over 12 hours by $17 \%$ compared with placebo and AP seemed to be most efficacious in the $60 \mathrm{mg}$ dose group, which is comparable in magnitude to those observed in previous studies of baclofen.

In this study, AP had a favorable tolerability and safety profile across the evaluated doses. There was no statistically significant difference in the number of patients experiencing adverse events after taking the active drug compared with placebo. Although the number of patients in this study was small, AP could be considered in proton pump inhibitor-refractory GERD patients in the near future.

\section{References}

1. Lal R, Sukbuntherng J, Tai EH, et al. Arbaclofen placarbil, a novel R-baclofen prodrug: improved ADME properties compared to R-baclofen. J Pharmacol Exp Ther 2009;330:911-921.

2. Gerson LB, Huff FJ, Hila A, et al. Arbaclofen placarbil decreases postprandial reflux in patients with gastroesophageal reflux disease. Am J Gastroenterol 2010;105:1266-1275.

3. Fass R, Gasiorowska A. Refractory GERD: what is it? Curr Gastroenterol Rep 2008;10:252-257.

4. Dent J, Dodds WJ, Friedman RH, et al. Mechanism of gastroesophageal reflux in recumbent asymptomatic human subjects. J Clin Invest 1980;65:256-267.

5. Babei A, Bhargava V, Korsapati H, Zheng WH, Mittal RK. A unique longitudinal muscle contraction pattern associated with transient lower esophageal sphincter relaxation. Gastroenterology 2008; 134:1322-1331.

6. Vela MF, Tutuian R, Katz PO, Castell DO. Baclofen decreases acid and non-acid post-prandial gastro-oesophageal reflux measured by combined multichannel intraluminal impedance and $\mathrm{pH}$. Aliment Pharmacol Ther 2003;17:243-251.

7. Ciccaglione AF, Marzio L. Effect of acute and chronic administration of the GABA B agonist baclofen on 24 hour $\mathrm{pH}$ metry and symptoms in control subjects and in patients with gastro-oesophageal reflux disease. Gut 2003;52:464-470.

8. Dario A, Tomei G. A benefit-risk assessment of baclofen in severe spinal spasticity. Drug Saf 2004;27:799-818.

9. Huff FJ, Lal R, Sukbuntherng J, et al. Clinical evaluation of XP19986 as a potential treatment for GERD. Am J Gastroenterol 2008; 103:S11. 